\title{
Therapeutic Effect of Gypenosides on Nonalcoholic Steatohepatitis via Regulating Hepatic Lipogenesis and Fatty Acid Oxidation
}

\author{
Hongshan Li, ${ }^{*, a, b}$ Hao Ying, ${ }^{a}$ Airong $\mathrm{Hu},{ }^{a}$ Yaoren $\mathrm{Hu},{ }^{a}$ and Dezhou $\mathrm{Li}^{a}$ \\ ${ }^{a}$ Department of Liver Disease, Ningbo No. 2 Hospital; Ningbo 315010, Zhejiang, China: and ${ }^{b}$ Medical School of \\ Ningbo University; Ningbo 315010, Zhejiang, China. \\ Received December 3, 2016; accepted February 14, 2017
}

Nonalcoholic steatohepatitis (NASH) is the most frequent cause of liver dysfunction and a common global problem. Gypenosides can decrease pathological modifications of high-fat diet-induced rat atherosclerosis; however, its effect and mechanism on NASH remain unclear. In this study, rats were randomly divided into normal control and model groups. Model rats were fed with a high-fat diet and treated with gypenosides, rosiglitazone, or water for 6 weeks. We found that liver tissues showed significant hepatic steatosis and vacuolar degeneration with significantly higher triglyceride (TG), free fatty acid (FFA) and malonyl CoA, serum alanine aminotransferase (ALT), aspartate aminotransferase (AST) and gamma-glutamyl transferase (GGT) activities in model group versus normal control group $(p<0.01)$. Liver tissue $\mathrm{mRNA}$ and protein levels of sterol regulatory element binding protein-1c (SREBP-1c), carbohydrate response element binding protein (ChREBP), acetyl-CoA carboxylase (ACCase), and stearoyl CoA desaturase enzyme 1 (SCD1) were significantly increased, while the carnitine palmitoyl transferase-1 (CPT-1) level was significantly decreased in the model group versus the normal control group $(p<0.01)$. Pathological changes of hepatic steatosis; body weight and liver wet weight; liver tissue TG, FFA and malonyl CoA concentrations; serum ALT, AST and GGT activities; liver tissue mRNA and protein levels of SREBP-1c, ChREBP, ACCase, and SCD-1 were significantly decreased; protein and mRNA levels of CPT-1 were significantly increased in the gypenosides group versus model group $(p<0.01)$. In conclusion, gypenosides has therapeutic effect on NASH through regulating key transcriptional factors and lipogenic enzymes involved in fatty acid oxidation during hepatic lipogenesis.

Key words gypenoside; nonalcoholic steatohepatitis; hepatic lipogenesis; fatty acid oxidation

Nonalcoholic steatohepatitis (NASH) is an important stage in the development of nonalcoholic fatty liver disease (NAFLD) and is one of the most frequent causes of liver dysfunction. NASH is also a key phase in the shift from simple steatosis to more severe hepatic diseases, such as hepatic fibrosis, liver cirrhosis, and hepatocellular carcinoma. ${ }^{1)}$ The epidemiological data demonstrate that the NAFLD occurrence rate is $25-30 \%$ in the U.S.A., $12.6-50 \%$ in Asia (Japan, China, and South Korea), and $3-10 \%$ in children, respectively. ${ }^{2-6)}$ Thus, NAFLD is an increasingly common global health problem, especially in the youth population, which is caused by changes in modern lifestyles and diets. ${ }^{7}$ NASH is presently the most rapidly growing indication for liver transplantation in hepatocellular carcinoma patients in the U.S.A., and it will become the leading indication for liver transplantation in the near future. ${ }^{8)}$

The mechanism of NASH progression has not been well explored, but it is thought to be related to different metabolic disorder factors, such as adipocyte factor, intestinal endotoxin disease, oxygen stress, and insulin resistance. ${ }^{9)}$ It is also known that inflammatory cytokines and oxidative stress play key roles in the pathogenesis and development of NASH, leading to the shift from a fatty liver to more severe conditions. ${ }^{10)}$ Many molecular pathways and factors are involved in the development of NASH, and the most important inflammatory factors include free fatty acids (FFAs). Under normal conditions, fatty acid $\beta$-oxidation in the mitochondria plays an important role in energy homoeostasis, and mitochondrial dysfunction results in a reduction of fatty acid $\beta$-oxidation, which is a critical feature of NAFLD. ${ }^{11)}$

NASH is characterized by intracellular triglyceride (TG) accumulation, liver fat deposition, inflammatory cell infiltration, ballooning of hepatocytes in the liver, and different degrees of fibrosis, ${ }^{12,13)}$ which result from an imbalance of increased lipids via circulating lipid uptake, de novo lipogenesis, or decreased lipid disposal primarily by FFA oxidation, and it is considered the first hit in the pathogenesis of NAFLD. The plasma FFA composition has been used to provide insight into cellular metabolic pathways. However, there are currently no efficient pharmaceutical therapeutics for NASH. ${ }^{14)}$ Therefore, the exploration of an appropriate pharmacological therapy for NASH is important.

Herbal treatment for NAFLD has received increasing attention in recent decades due to its wide availability, minimal side effects, and proven therapeutic mechanisms and benefits. ${ }^{6}$ Evidence-based medicine supports this point of view. Gynostemma pentaphyllum (THUNB.) MAKINO is a cucurbitaceous herbaceous vine that is widely used in traditional medicine in China and other Asian countries for the treatment of cancer, hyperlipoproteinemia, hepatitis, and cardiovascular disease. ${ }^{15)}$

Gypenosides, the major active component extract from $G$. pentaphyllum (Thunb.) MAKINO, exist mainly as dammaranetype triterpene glycosides and possess a number of pharmacological properties, including anti-aging, cardioprotective, and neuroprotective effects. $\left.{ }^{16}\right)$ Gypenosides also have been shown to enhance superoxide dismutase (SOD) activity in serum and tissues, decrease serum levels of oxidized low-density lipoprotein, and increase total antioxidant ability. However, there is no information about the protective effect of gypenosides on NASH, especially the regulatory mechanism on liver fat metabolism.

In this study, for the first time, we provide compelling 
evidence of the therapeutic efficacy of gypenosides on high-fat diet-induced NASH and the potential mechanisms involved in liver fat metabolism.

\section{MATERIALS AND METHODS}

Test Drug and Reagents Gypenosides (lot no.: ZL150307100; Specification: 98\%; yield: 60:1) was purchased from Nanjing Zelang Medical Technology Co., Ltd.; rosiglitazone (lot no.: 150109) was purchased from Chengdu Hengrui Pharmaceutical Co., Ltd. (Chengdu, China); the high-fat diet was produced by Shanghai Puluteng Biotech Co., Ltd. (Shanghai, China); TG (lot no.: 20151029), FFA (lot no.: 20151030), aspartate aminotransferase (AST, lot no.: 20151028), and gamma-glutamyl transferase (GGT, lot no.: 20151029) detection kits were purchased from Nanjing Jiancheng Biological Engineering Institute (Nanjing, China); sterol regulatory element binding protein-1c (SREBP-1c, lot no.: 201509), carbohydrate response element binding protein (ChREBP, lot no.: 201510), acetyl CoA carboxylase (ACCase, lot no.: 201511), stearoyl CoA desaturase 1 (SCD1, lot no.: 201603), (malonyl CoA, lot no.: 201510), and carnitine palmitoyl transferase-1 (CPT-1, lot no.: 201510) enzyme-linked immunoassay (ELISA) kits were purchased from Shanghai Yeyuan Biotechnology Company (Shanghai, China); the RNAiso Plus kit (lot no. AK9108), PrimeScript $^{\mathrm{TM}}$ RT Master Mix (Perfect Real Time) (lot no.: AK4403) and $\mathrm{SYBR}^{\circledR}$ Premix Ex $\mathrm{Taq}^{\mathrm{TM}}$ (Tli RNaseH Plus) (lot no.: AK7501) were obtained from TaKaRa Holdings Inc. (Kusatsu, Japan).

In Vivo High-Fat Diet-Induced NASH Model Establishment and Treatment A total of 32 clean grade, male Sprague Dawley rats (body weight, 100-120 g) were purchased from Shanghai SLAC Laboratory Animal Co., Ltd. (Shanghai, China). Rats were housed at the Animal Experiment Center of Ningbo University and had free access to water. The laboratory animal certificate number was SCXK (Shanghai) 2012-0002. All procedures and animal experiments were approved by the Animal Care and Use Committee, Ningbo University. The rats were randomly divided into two groups, with 8 in the normal control group and 24 in the model group, using a random number table just prior to the experiment.

For preparation of the NASH model, rats in the model group were fed with mixed food, which had been sanitized by cobalt 60 irradiation and contained $88 \%$ basal food, $10 \%$ lard, and $2 \%$ cholesterol, for 14 weeks, after adaptive feeding for 1 week. ${ }^{17)}$ Rats in the normal control group were only fed with normal basal food.

It has been reported that rosiglitazone alone shows convincing therapeutic efficacy in $\mathrm{NASH},{ }^{18)}$ and it has been used as positive control previously. ${ }^{19)}$ Therefore, in the current study, rats treated with rosiglitazone alone were used as a positive control.

From the 9th week of the high-fat diet, rats in the model group were randomly separated into the model group, gypenosides group, and rosiglitazone group $(n=8)$, using a random number table. Gypenosides $(11.49 \mathrm{mg} / \mathrm{kg}$ body weight $/ \mathrm{d})$ and rosiglitazone $(0.4 \mathrm{mg} / \mathrm{kg}$ body weight $/ \mathrm{d})$ were respectively given to the rats by intragastric gavage administration for 6 continuous weeks, while an equal volume of drinking water was administered by the same method to the rats in the normal control and model groups.
Determination of the Dose of Gypenosides and Rosiglitazone The daily rat dosages of gypenosides and rosiglitazone used in our current study were calculated according to the conversion formula between humans and rats $(1: 6)$. The daily human dosage of gypenosides was determined by the middle human daily dosage $(6.5 \mathrm{~g})$ of Gynostemma pentaphylla recommended in the Pharmacopoeia of the People's Republic of China, and the content of gypenosides in G. pentaphylla, which indicated that $6.5 \mathrm{~g}$ of $G$. pentaphylla contained about $110.54 \mathrm{mg}$ of gypenosides. The daily human dosage, $4 \mathrm{mg} / \mathrm{d}$ for a $60-\mathrm{kg}$ (body weight) adult, of rosiglitazone was determined by the recommended dosage by the manufacturer of Chengdu Hengrui Pharmaceutical Co., Ltd.

Specimen Collection After 6 weeks of treatment, the rats were anesthetized with $2 \%$ sodium pentobarbital $(3 \mathrm{~mL} / \mathrm{kg})$ by intraperitoneal injection after fasting for $12 \mathrm{~h}$. Then, the rat abdominal cavities were opened and blood was drawn from the inferior vena cava. The serum was collected by centrifugation at $4^{\circ} \mathrm{C}$ and stored at $-70^{\circ} \mathrm{C}$. Liver tissues were isolated and cut into two pieces: one piece was fixed in $10 \%$ neutral buffered formalin for hematoxylin \& eosin (H\&E) staining, and the other piece was stored at $-80^{\circ} \mathrm{C}$ for RNA and protein extraction. Body and liver weights of the rats were measured for the calculation of the liver weight index, according to the following equation: liver weight index $=$ (liver weight $/$ body weight) $\times 100 \%$.

Histopathological Staining Rat liver tissues (approximately $1 \times 1 \times 0.5 \mathrm{~cm}$ ) were dissected and fixed in $10 \%$ neutral buffered formalin for $24 \mathrm{~h}$, then washed with sterile $\mathrm{H}_{2} \mathrm{O}$, and stored in $70 \%$ ethanol. Liver tissues were embedded in paraffin, and sections (4- $\mu \mathrm{m}$ thick) were stained with HE staining solution to visualize the morphology. Slices were observed, and the degree of hepatic steatosis $\left(\mathrm{F}_{0}-\mathrm{F}_{4}\right)$ was determined according to the guidelines for the management of $\mathrm{NFLD}^{20)}$ in each sample under a light microscope (Olympus BX50). Images were obtained by an image autoanalysis system, and one representative area is shown.

Analysis of Hepatic TG and FFA Hepatic TG and FFA concentrations were determined according to the manufacturer's descriptions or references. A total of $3 \mathrm{~mL}$ of ethanolacetone $(1: 1)$ was added to $200 \mathrm{mg}$ (wet weight) of liver tissue from each rat, and the tissue was homogenized at $3000 \mathrm{rpm}$, three times $10 \mathrm{~s}$, using a Bio-Gen PRO200 homogenizer. The homogenate was mixed well and kept on ice overnight before being centrifuged at $4^{\circ} \mathrm{C}$ and $3000 \mathrm{rpm}$ for $15 \mathrm{~min}$. The supernatant was divided into $1.5-\mathrm{mL}$ centrifuge tubes for the $\mathrm{TG}$ concentration assay, according to the manufacturer's descriptions. In brief, $10 \mu \mathrm{L}$ of distilled water was added to the blank tube, $10 \mu \mathrm{L}$ of $2.26 \mathrm{~mm}$ calibrator was added to the standard quality control tube, and $10 \mu \mathrm{L}$ of sample was added to the sample tube. A total of $1000 \mu \mathrm{L}$ of working fluid was added to each tube above, and the solution was gently mixed. Then, all the tubes were kept at $37^{\circ} \mathrm{C}$ in a warm bath for $10 \mathrm{~min}$, and the optical density (OD) values were determined at a wavelength of $510 \mathrm{~nm}$.

A total of $1 \mathrm{~mL}$ of phosphate-buffered saline was added to $100 \mathrm{mg}$ (wet weight) of liver tissue from each rat, and the tissue was homogenized at $10000 \mathrm{rpm}$ on ice, three times $20 \mathrm{~s}$, using a Bio-Gen PRO200 homogenizer. The homogenate was centrifuged at $4^{\circ} \mathrm{C}$ and $3600 \mathrm{rpm}$ for $20 \mathrm{~min}$. The supernatant was collected and used for the FFA concentration assay, ac- 
cording to a method reported previously. ${ }^{21)}$

Serum ALT, AST, and GGT Activity Assay Serum ALT, AST, and GGT activity assays were performed, according to the manufacturers' descriptions. In brief, $20 \mu \mathrm{L}$ of substrate solution was added to each of the control and assay wells in a 96-well plate, and then $5 \mu \mathrm{L}$ of each sample to be tested was added to the sample well and mixed. The plate was incubated at $37^{\circ} \mathrm{C}$ for $30 \mathrm{~min}, 200 \mu \mathrm{L}$ of $0.4 \mathrm{M}$ sodium hydroxide solution was added to each well, and then the plate was gently shaken for mixing and incubated at room temperature for $15 \mathrm{~min}$. The OD values were determined at a wavelength of $510 \mathrm{~nm}$ using a microplate reader.

RNA Extraction and Real-Time PCR Analysis Total RNA was extracted from homogenized hepatic tissue samples using an RNAiso Plus kit. The OD values at $260 \mathrm{~nm}$ were measured using a NanoDrop 1000 spectrophotometer (Thermo Fisher Scientific, U.S.A.) to determine the RNA concentrations. The PCR primers were designed by Shanghai Han Yue Biological Technology Co., Ltd. (Shanghai, China) and synthesized by Shanghai Biological Technology Co., Ltd. (Shanghai, China). The primers used were as follows: ACCase, 5'-TTT GTG ATG ACT TTG AGG GA-3' (forward) and 5'-GGA GAGGAACTGAAC TGT GC-3' (reverse); SCD-1, 5'- CGCCAC TGACTTGCT ATG TT-3' (forward) and 5'-CAGGAGGTTCTTGGGATGAT-3' (reverse); ChREBP, 5'-CTGAGGGAT GAAATA GAGG-3' (forward) and 5'-ATA AAGGTCGGA TGAGGAT-3' (reverse); SREBP1c, 5'-TCC TGC ACC ACC AAC TGCTTAG-3' (forward) and 5'-AGT GGC AGT GAT GGC ATGGAC T-3' (reverse); and CPT-1, 5'-CTTCCCCTT ACT GGT TCC-3' (forward) and 5'-ACTCTCCCGCTGTTG TCC-3' (reverse). Reverse transcription was done using a PrimeScript ${ }^{\text {TM }}$ RT Master Mix (Perfect Real Time) kit. Real-time PCR was performed using quantitative PCR reagents $\mathrm{SYBR}^{\circledR}$ Premix Ex Taq ${ }^{\mathrm{TM}}$ II and the 7900 HT Sequence Detection System (ABI, U.S.A.). Glyceraldehyde-3-phosphate dehydrogenase (GAPDH) levels were used as internal controls, and fold changes were calculated using the $2^{-\Delta \Delta C t}$ method. Each experiment was performed in triplicate.

ELISA Liver tissue homogenate was prepared as described above for the FFA concentration assay. SREBP-1c, ChREBP, ACCase, SCD1, malonyl CoA, and CPT-1 in the liver tissue homogenate were measured using ELISA kits, according to the manufacturers' instructions. In brief, $50 \mu \mathrm{L}$ of diluted standard substance was added to a 96-well plate to prepare a standard curve. Next, $50 \mu \mathrm{L}$ of each sample was added to the indicated wells. After five washes, $50 \mu \mathrm{L}$ of enzyme-labelled reagent was added to each well, except for the blank well. The plate was then incubated at $37^{\circ} \mathrm{C}$ for $30 \mathrm{~min}$. Next, $50 \mu \mathrm{L}$ of color development reagents $\mathrm{A}$ and $\mathrm{B}$ were added to each well, and the plate was incubated at $37^{\circ} \mathrm{C}$ for $15 \mathrm{~min}$ in the dark. Finally, the OD value was determined at $450 \mathrm{~nm}$ using a CYTATION 3 microplate reader (BioTek, U.S.A.) within $15 \mathrm{~min}$ after adding $50 \mu \mathrm{L}$ of stop buffer.

Statistical Analyses All statistical analyses were performed with SPSS16.0 software. Measurement data were presented as the mean \pm standard deviation (S.D.). Data between groups were compared using one-way ANOVA as well as the least significant difference test, and a value of $p<0.05$ was considered to be statistically significant.
Table 1. Changes of Rat Body Weight, Liver Wet Weight, and Liver Weight Index (Mean \pm S.D.)

\begin{tabular}{llccc}
\hline \hline \multicolumn{1}{c}{ Group } & $n$ & $\begin{array}{c}\text { Body weight } \\
(\mathrm{g})\end{array}$ & $\begin{array}{c}\text { Liver wet weight Liver weight index } \\
(\mathrm{g})\end{array}$ & \multicolumn{1}{c}{$(\%)$} \\
\hline Normal & 8 & $537 \pm 60^{* *}$ & $13.43 \pm 1.89 * *$ & $2.53 \pm 0.47 * *$ \\
Model & 8 & $658 \pm 47$ & $25.36 \pm 6.09$ & $3.88 \pm 1.05$ \\
Gypenosides & 8 & $587 \pm 47 *$ & $18.84 \pm 3.71 *$ & $3.22 \pm 0.68$ \\
Rosiglitazone & 8 & $575 \pm 39 * *$ & $19.03 \pm 2.58 *$ & $3.32 \pm 0.48$ \\
\hline
\end{tabular}

Quantitative data are expressed as the mean \pm S.D. Data between groups were compared using one-way ANOVA as well as the least significant difference test. $* * p<0.01, * p<0.05, v s$. the model group.

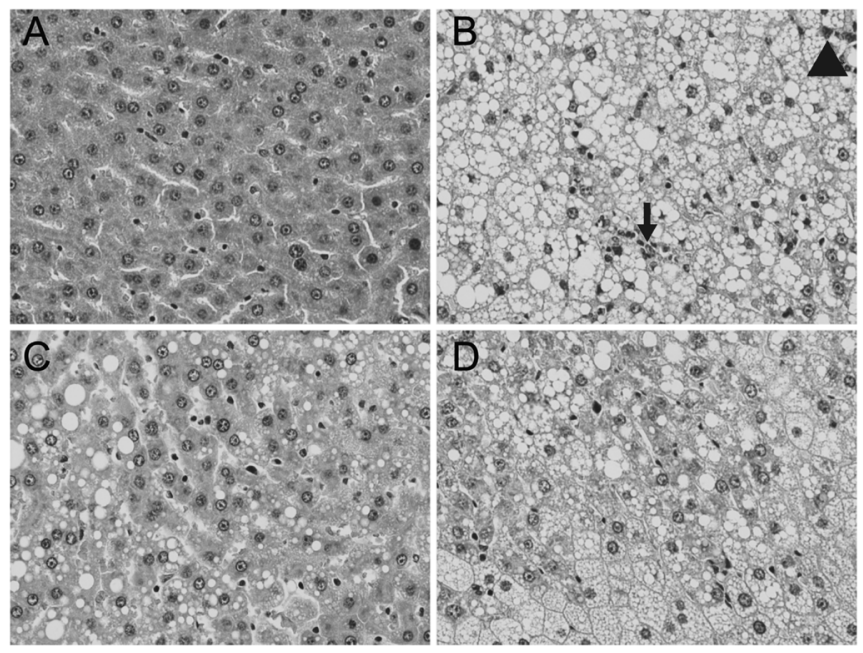

Fig. 1. Histological Changes of Liver Tissue Sections in Different Groups $(\times 400)$

HE staining of the liver tissue sections of each group: (A) normal control group; (B) model group; (C) gypenosides group; and (D) rosiglitazone group. The infiltration of inflammatory cells and spotty necrosis on histopathological changes have been marked with arrows.

\section{RESULTS}

Rat Body Weight, Liver Weight, and Liver Weight Index Comparison After the indicated medication cycle of 6 weeks, the rats were anesthetized and the liver tissues were collected. The body weight and liver wet weight of each rat were measured, and the liver weight index was calculated according to the equation described in the Materials and Methods. As shown in Table 1, the rat body weight, liver wet weight, and liver weight index in the model group were all significantly increased compared to the control (all $p<0.01$ ). The rat body weight and liver wet weight in both the gypenosides and rosiglitazone groups were significantly lower than those in the model group $(p<0.05)$; and the liver weight indexes in the gypenosides and rosiglitazone groups were decreased compared to the model group, but the difference was not statistically significant $(p>0.05)$. Moreover, the rat body weight, liver wet weight, and liver weight index showed no significant difference between the gypenosides group and the rosiglitazone group $(p<0.05)$.

Histopathological Changes of Rat Liver Tissues Rat liver tissue sections were stained with HE. As shown in Fig. 1, in the normal control group, the hepatic lobule and rope both showed clear structures and a regular arrangement. There was no point necrosis or lobular inflammatory cell infiltration. The hepatocyte morphologies were normal, the hepatocyte nuclei 
were blue, and the cytoplasm was stained uniformly red with little or no adipose hollow spaces. Rat hepatic steatosis was significant in the model group, and the portal area and central vein showed diffuse adipose hollow spaces. The hepatocytes showed obvious swelling or enlargement, the cytoplasm was loose with more visible fat droplet accumulation, the nuclei were drifted in some cells, and mild infiltration of inflammatory cells and scattered spotty necrosis were found in the model group. Compared with the model group, the structure arrangement, morphological characteristics, adipose hollow space, inflammatory cell infiltration, and spotty necrosis were all improved in the gypenosides and rosiglitazone treatment groups.

According to the diagnostic guidelines of NAFLD, liver steatosis is divided into four degrees $\left(\mathrm{F}_{0}-\mathrm{F}_{4}\right)$ as follows: $\mathrm{F}_{0}$, $<5 \%$ hepatocellular steatosis; $\mathrm{F}_{1}, 5-30 \%$ hepatocellular steatosis; $\mathrm{F}_{2}, 31-50 \%$ hepatocellular steatosis; $\mathrm{F}_{3}, 51-75 \%$ hepatocellular steatosis; and $\mathrm{F}_{4},>75 \%$ liver cell steatosis. Ridit analysis showed that, compared with the normal control group, the degree of hepatic steatosis was significantly higher in the model group $(p<0.01)$. In addition, the degree of hepatic steatosis of the gypenosides and rosiglitazone groups was significantly decreased, compared with the model group $(p<0.05)$. Moreover, the degree of hepatic steatosis in the gypenosides group

Table 2. Comparison of the Degree $\left(\mathrm{F}_{0}\right.$ to $\left.\mathrm{F}_{4}\right)$ of Rat Hepatic Steatosis in HE-Stained Liver Tissues from the Different Groups

\begin{tabular}{lcccccc}
\hline \multirow{2}{*}{ Group } & \multicolumn{5}{c}{ Degree of hepatic steatosis } & \multirow{2}{*}{ Total } \\
\cline { 2 - 6 } & $\mathrm{F}_{0}$ & $\mathrm{~F}_{1}$ & $\mathrm{~F}_{2}$ & $\mathrm{~F}_{3}$ & $\mathrm{~F}_{4}$ & \\
\hline Normal** & 8 & 0 & 0 & 0 & 0 & 8 \\
Model & 0 & 0 & 0 & 5 & 3 & 8 \\
Gypenosides** & 0 & 1 & 5 & 2 & 0 & 8 \\
Rosiglitazone** & 0 & 0 & 3 & 4 & 1 & 8 \\
Total & 8 & 1 & 8 & 11 & 4 & 32 \\
Ridit value & 0.1250 & 0.2656 & 0.4062 & 0.7031 & 0.9375 & \\
\hline
\end{tabular}

Data between groups were compared using one-way ANOVA as well as the leas significant difference test. ${ }^{* *} p<0.01,{ }^{*} p<0.05$, vs. the model group; ${ }^{*} p<0.05$, vs. the gypenosides group. According to the diagnostic guidelines of NAFLD, liver steatosis is divided into four degrees $\left(\mathrm{F}_{0}-\mathrm{F}_{4}\right)$ as follows: $\mathrm{F}_{0},<5 \%$ hepatocellular steatosis; $\mathrm{F}_{1}$, $5-30 \%$ hepatocellular steatosis; $F_{2}, 31-50 \%$ hepatocellular steatosis; $F_{3}, 51-75 \%$ hepatocellular steatosis; and $\mathrm{F}_{4},>75 \%$ liver cell steatosis. was significantly lower than that of the rosiglitazone group $(p<0.05)$ (Table 2).

Changes of Rat Hepatic TG and FFA Hepatic TG and FFA concentrations were determined in the liver tissue homogenates after the rats were treated with the indicated medication or vehicle for 6 weeks. As shown in Figs. 2A and B, the TG and FFA concentrations in the rat liver tissues in the model group were drastically higher than those in the normal control group $(p<0.01)$, while both of them were significantly reduced in the gypenosides group and rosiglitazone group, compared with the model group $(p<0.01)$. Furthermore, the liver tissue TG and FFA concentrations in the gypenosides group were obviously lower than those in the rosiglitazone group $(p<0.01)$. These findings suggest that gypenosides can modulate lipid metabolism and accumulation in the livers of NASH rat models.

Changes of Rat Serum ALT, AST, and GGT Activities To evaluate the liver function injury caused in NASH rats and the potential effect of gypenosides, the serum ALT (Fig. 3A), AST (Fig. 3B), and GGT (Fig. 3C) activities were determined. The results showed that compared with the normal control group, the serum ALT, AST, and GGT activities were all significantly increased in the model rats $(p<0.01)$. Meanwhile, the serum ALT, AST, and GGT activities in the gypenosides group and the ALT activity in the rosiglitazone group were decreased significantly, compared with the model group $(p<0.05)$. However, the serum AST and GGT activities in the rosiglitazone group showed no significant difference compared to those in the model group $(p>0.05)$. In addition, the serum AST and GGT activities in the gypenosides group were all significantly lower than those in the rosiglitazone group $(p<0.05)$. But the serum ALT activity in the gypenosides group was not significantly different than that of the rosiglitazone group $(p>0.05)$. These findings suggest that gypenosides have a protective effect on the liver function of NASH rat models.

Changes of Rat Hepatic SREBP-1c, ChREBP, ACCase, SCD-1, Malonyl CoA, and CPT-1 Protein Concentrations To explore the molecular mechanism of gypenosides in the treatment of NASH, we detected and compared the SREBP-1c, ChREBP, ACCase, SCD-1, malonyl CoA, and CPT-1 protein
A

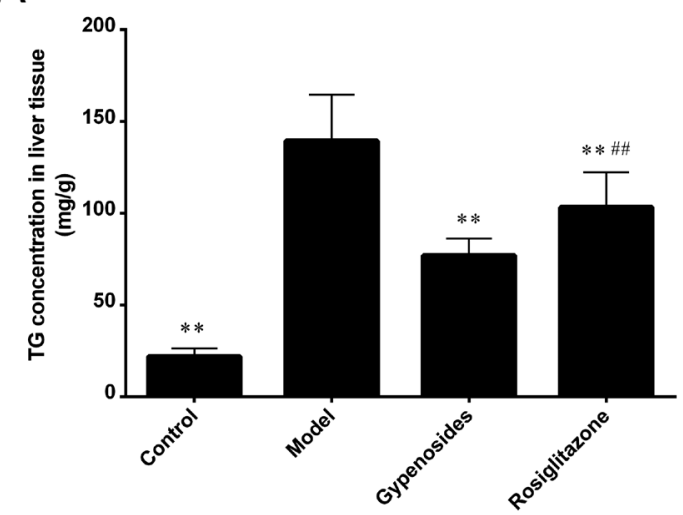

B

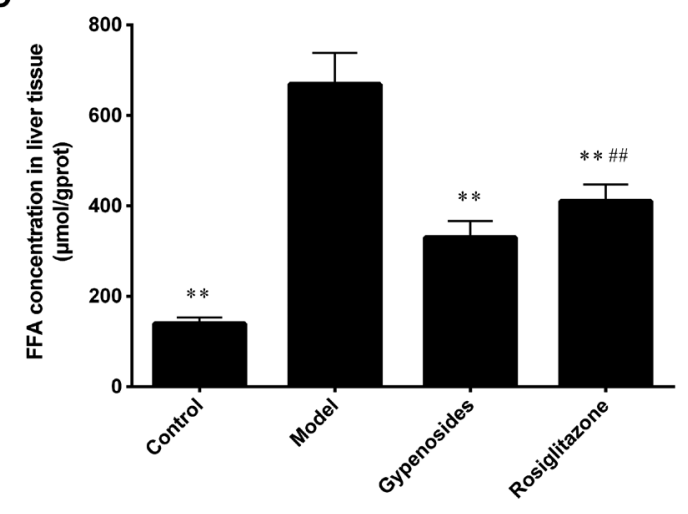

Fig. 2. Changes of Rat Hepatic TG and FFA

Hepatic TG and FFA concentrations were determined in the liver tissue homogenates after rats were fed with high-fat diet foods, except for the normal control group, which was fed with normal foods, and treated with the indicated medication or vehicle for 6 weeks. (A) TG concentration in liver tissue homogenate; (B) FFA concentration in liver tissue homogenate. Data between groups were compared using one-way analysis of variance as well as the least significant difference test. $* * p<0.01, v e r s u s$ the model group; ${ }^{\# \#} p<0.01$, versus the gypenosides group. 
A

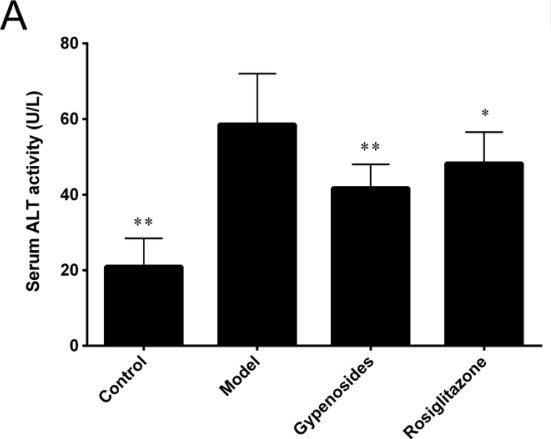

B

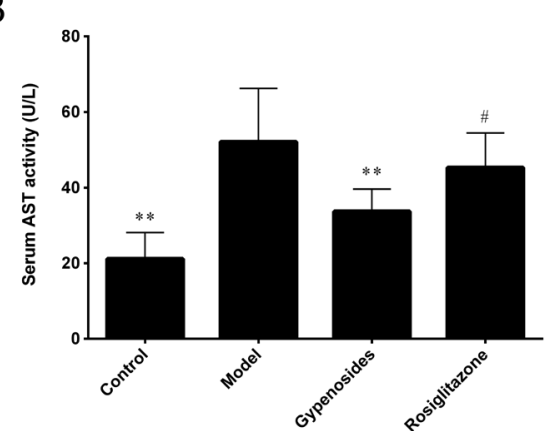

C

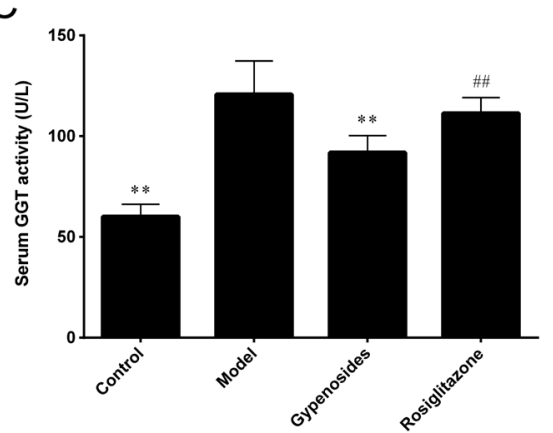

Fig. 3. Changes of Rat Serum Liver Function Indexes

Serum was collected from rats of each group, and ALT, AST, and GGT activities were determined, respectively. (A) ALT activity; (B) AST activity; and (C) GGT activity. ${ }^{*} p<0.05,{ }^{*} p<0.01$, versus the model group. Data between groups were compared using one-way analysis of variance as well as the least significant difference test. ${ }^{\#} p<0.05,{ }^{\#} p<0.01$, versus the gypenosides group.

concentrations in the liver tissue homogenates of NASH rat models with or without gypenosides treatment. As shown in Fig. 4, the SREBP-1c, ChREBP, ACCase, SCD-1, and malonyl $\mathrm{CoA}$ protein concentrations were significantly increased in the liver tissue of the model group, compared to those of the normal control group $(p<0.01)$; and the protein concentration of CPT-1 was significantly decreased in the liver tissue of the model group, compared to that of the normal control group $(p<0.01)$. In addition, the SREBP-1c, ChREBP, ACCase, SCD-1, and malonyl CoA protein concentrations in the liver tissues of the gypenoside group as well as the SREBP-1c and ChREBP protein concentrations in the liver tissues of the rosiglitazone group were significantly decreased, compared with those of the model group $(p<0.01)$. The protein concentration of CPT-1 was significantly increased in the liver tissue of the rosiglitazone group, compared to that of the model group $(p<0.01)$. Meanwhile, the ACCase, SCD-1, and malonyl CoA protein concentrations in the liver tissues of the rosiglitazone group were not significantly different from those of the model group ( $p>0.05$ ). The SREBP-1c, ACCase, SCD-1 and malonyl CoA protein concentrations were significantly decreased in the liver tissues of the gypenosides group, compared to those in the rosiglitazone group $(p<0.05)$. However, the ChREBP and CPT-1 protein concentrations in the liver tissues of the gypenosides group were not significantly difference from those in the rosiglitazone group $(p>0.05)$. These data indicate that SREBP-1c, ChREBP, ACCase, SCD-1, malonyl CoA, and CPT-1 are closely implicated in the anti-NASH effect of gypenosides in rat models. Moreover, these data demonstrate that gypenosides exert an anti-NASH effect by regulating the SREBP-1c, ChREBP, ACCase, SCD-1, malonyl CoA, and CPT-1 protein levels in liver tissues.

Changes of Rat Hepatic SREBP-1c, ChREBP, ACCase, SCD-1, and CPT-1 mRNA Expression Levels To identify whether the key factors described above that are involved in the anti-NASH effect of gypenosides in rat models also elicit the same changes at the transcriptional level, we further determined the mRNA expression levels of SREBP-1c, ChREBP, ACCase, SCD-1, and CPT-1 in liver tissue homogenates of each group by real-time PCR analysis. As shown in Fig. 5, the mRNA expression levels of SREBP-1c, ChREBP, ACCase, and SCD-1 were drastically increased and that of CPT-1 was significantly decreased in the rat liver tissues of the model group, compared to the normal control group $(p<0.01)$. Moreover, the SREBP-1c, ChREBP, ACCase, and SCD-1 mRNA expression levels in the liver tissues of the gypenosides group were obviously decreased while that of CPT-1 was significantly increased, compared to those of the model group $(p<0.01)$. Furthermore, the SREBP-1c, ACCase, and SCD-1 mRNA expression levels in the liver tissues of the rosiglitazone group were considerably decreased while that of CPT-1 was significantly increased, compared to those of the model group $(p<0.05)$. However, the ChREBP mRNA expression in the liver tissues of the rosiglitazone group was not significantly different from that of the model group $(p>0.05)$. The SREBP1c, ChREBP, and SCD-1 mRNA expression levels were significantly decreased in the liver tissues of the gypenosides group, compared to those of the rosiglitazone group $(p<0.01)$. Meanwhile the ACCase and CPT-1 mRNA expression levels in liver tissues of the gypenosides group were not significantly different from those in the rosiglitazone group $(p>0.05)$. Our data further confirmed that the regulation of SREBP-1c, ChREBP, ACCase, SCD-1, and CPT-1 at the transcriptional level in liver tissues was involved in the therapeutic effect of gypenosides.

\section{DISCUSSION}

It is well known that the initiation and development of NAFLD is closely associated with an increased visceral fat volume as well as hepatocytic accumulation of FFAs and $\mathrm{TG}^{22)}$ which are relatively common in obese NAFLD patients. TGs are derived from the esterification of FFAs that may be from adipose tissues or dietary fats. Lipolysis of hepatic TGs is mediated by lipases, which release FFA for oxidation. After synthesis, hepatic TGs may be stored as lipid droplets or secreted into the circulation packaged with apolipoprotein B into very-low-density lipoprotein. ${ }^{23)}$

In this study, by using a high-fat diet-induced rat NASH model, significantly increased body weight, liver wet weight, and liver weight index; hepatic TG and FFA concentrations; serum ALT, AST, and GGT activities as well as diffuse adipose hollow space, obvious swelling or enlargement of hepatocytes, more visible fat droplet accumulation in the cytoplasm, mild infiltration of inflammatory cells, and scattered spotty necrosis were observed in model group rats; however, this situation was drastically improved by gypenosides treatment, which is evidenced by a statistically significant decrease in rat body weight, liver wet weight, liver tissue TG and FFA concentrations, serum ALT, AST, and GGT activities, adipose hollow space, inflammatory cell infiltration, and spotty 
A

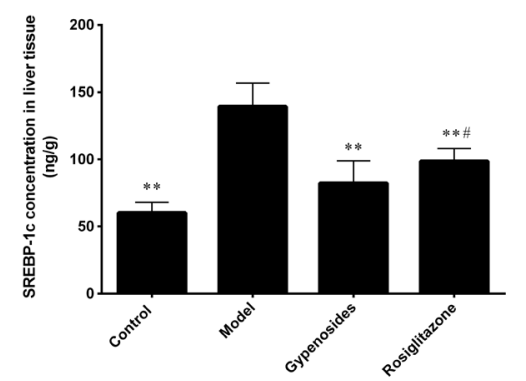

D

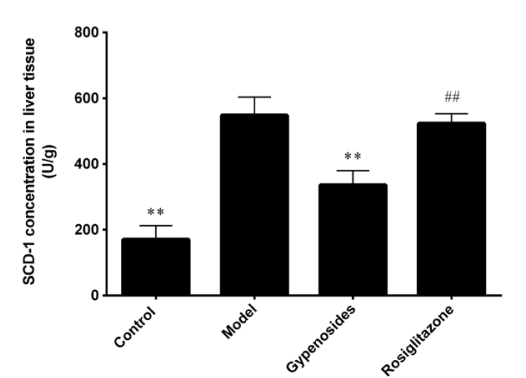

B

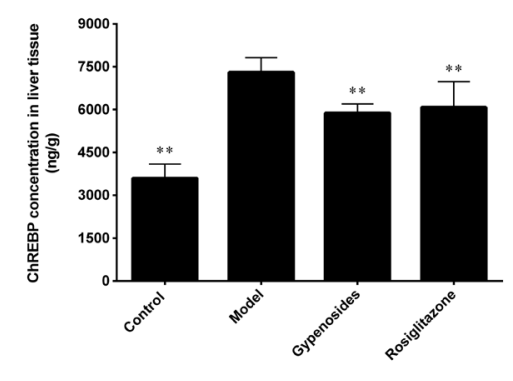

E

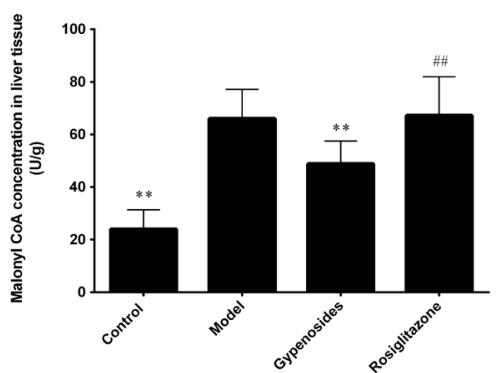

C

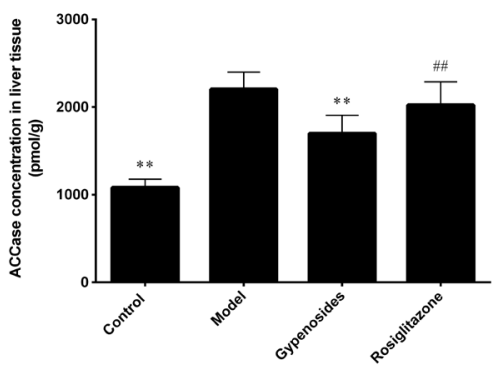

F

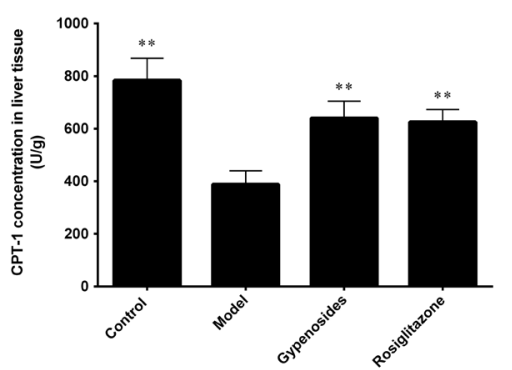

Fig. 4. Changes of Rat Hepatic SREBP-1c, ChREBP, ACCase, SCD-1, Malonyl CoA, and CPT-1 Protein Concentrations

Liver tissues were collected from rats of each group, and homogenates were prepared using a homogenizer. Changes of rat hepatic SREBP-1c, ChREBP, ACCase, SCD-1, malonyl CoA, and CPT-1 protein expression levels in the liver tissue homogenates were measured by ELISA, respectively. (A) SREBP-1c; (B) ChREBP; (C) ACCase; (D) SCD-1; (E) malonyl CoA; and (F) CPT-1. Data between groups were compared using one-way analysis of variance as well as the least significant difference test. $* * p<0.01$, versus the model group; ${ }^{\#} p<0.01$, versus the gypenosides group.

A

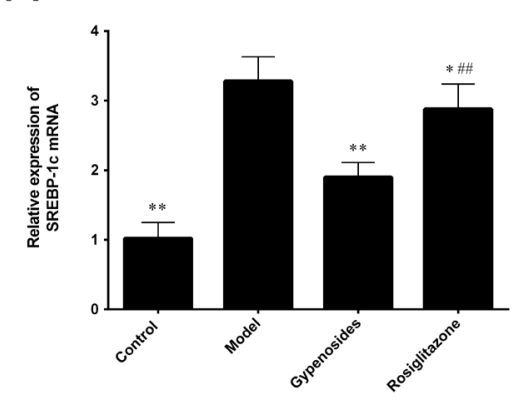

D

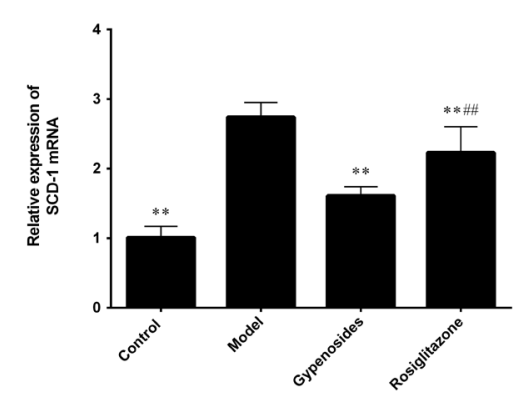

B

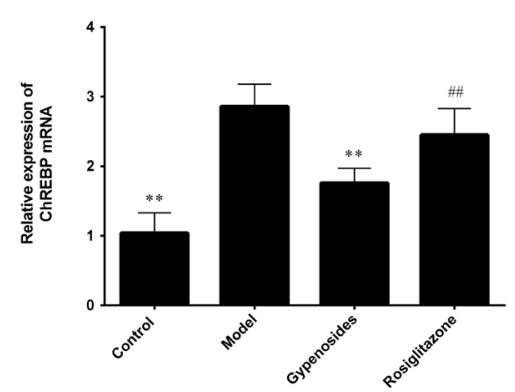

E
C

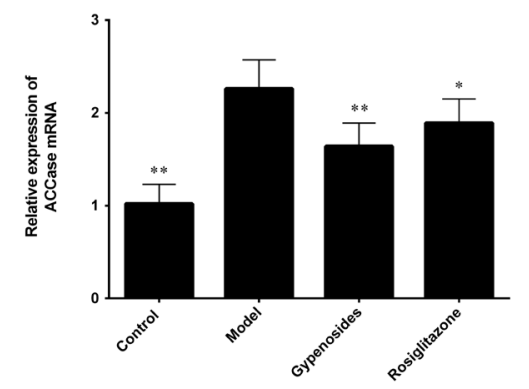

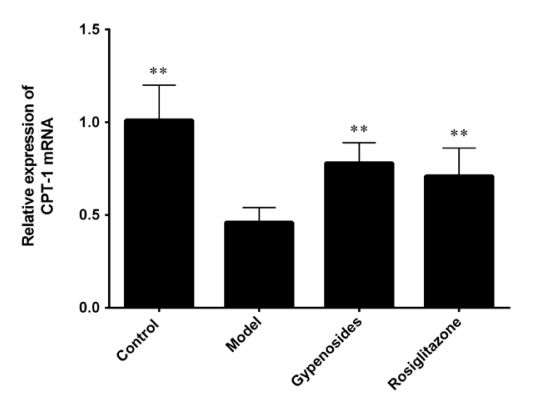

Fig. 5. Changes of Rat Hepatic SREBP-1c, ChREBP, ACCase, SCD-1, and CPT-1 mRNA Expression Levels

Liver tissues were collected from rats of each group, and homogenates were prepared using a homogenizer. Total RNA was extracted from the homogenates, and the mRNA expression levels of SREBP-1c, ChREBP, ACCase, SCD-1, and CPT-1 were determined by real-time PCR analysis. (A) SREBP-1c; (B) ChREBP; (C) ACCase; (D) SCD-1; and (E) CPT-1. Representative data shown are from three independent experiments with comparable results. Data between groups were compared using one-way analysis of variance as well as the least significant difference test. ${ }^{*} p<0.05,{ }^{*} p<0.01$, versus the model group; ${ }^{\# \#} p<0.01$, versus the gypenosides group.

necrosis after continuous gypenosides administration for 6 weeks in high-fat diet-induced rat NASH models. Rat hepatic TG and FFA concentrations with gypenosides treatment were only 55.20 and $49.33 \%$ of those without gypenosides treat- ment, respectively. Taken together, these data demonstrate that gypenosides treatment reduced body fat stores in rats fed with a high-fat diet and showed a potential pharmacodynamic effect in the prevention of NASH. Attractively, this effect was 
even better than that of rosiglitazone.

To explore the possible mechanisms for the decrease in serum and liver lipids caused by gypenosides treatment, we further investigated the expression levels of the key transcriptional factors and lipogenic enzymes involved in lipogenesis and fatty acid oxidation in liver tissues.

The present study showed that both the mRNA and protein levels of SREBP-1c and ChREBP in the liver tissue homogenates of rat NASH models were significantly increased and were statistically rescued by continuous gypenosides administration for 6 weeks in high-fat diet-induced rat NASH models. SREBP-1c is abundantly expressed in hepatocytes and is the key transcriptional factor that regulates the expression of genes required for hepatic fatty acid synthesis, such as SCD-1, ACCase, and lipogenic enzymes, and persistent activation of SREBP-1c is involved in the development of fatty liver disease and dyslipidemia. ${ }^{24-26)}$ In the livers of NAFLD patients, the expression of SREBP-1c and its target genes is significantly increased. ${ }^{27)} \mathrm{ChREBP}$ is a transcriptional activator of lipogenic and glycolytic genes and has emerged as a key regulator to control hepatic de novo fatty acid synthesis under physiological conditions as well as in NAFLD. ${ }^{28,29)}$ Target genes of ChREBP play important roles in glucose and lipid metabolism. ${ }^{30)}$ SREBP-1C and ChREBP synergistically activate the activation of several lipogenic enzymes, such as ACCase, malonyl CoA, SCD-1, and CPT-1, which are involved in energy storage through FFA and TG synthesis, resulting in increased FFA synthesis. ${ }^{31)}$

Our data further determined that the ACCase, SCD-1, and malonyl CoA protein levels as well as the ACCase and SCD-1 mRNA levels were drastically increased in the liver tissue homogenates of rat NASH models, showing a statistically significant decrease after continuous gypenosides administration for 6 weeks in high-fat diet-induced rat NASH models. In addition, both the protein and mRNA levels of CPT-1 were significantly increased in the gypenosides group, compared to the model group. ACCase is the rate-limiting enzyme during fatty acid synthesis, catalyzes the key step of de novo fatty acid biosynthesis, ${ }^{32)}$ and can be transformed into malonyl-CoA by acetyl-CoA carboxylase. ${ }^{33)}$ Malonyl-CoA is a key physiological regulator of both fatty acid synthesis and oxidation; it is also an intermediate in fatty acid synthesis and an allosteric inhibitor of CPT-1. ${ }^{34)}$ SCD-1 is the rate-limiting enzyme in the synthesis of monounsaturated fatty acids that converts palmitoyl- and stearoyl-CoA to palmitoleoyl and oleoyl-CoA, respectively. ${ }^{35)}$ The monounsaturated products of SCD-1 are preferred substrates for TG synthesis. ${ }^{35)} \mathrm{SCD}-1$ has been reported to be a direct target gene of SREBP- $1 \mathrm{c}^{36)}$ and is regulated by dietary and hormonal factors, including polyunsaturated fatty acids, cholesterol, insulin, fructose, and glucose. ${ }^{37)}$ As the key rate-limiting enzyme of fatty acid oxidation, CPT-1 controls fatty acid oxidation directly and catalyzes the formation of acyl-carnitine from acyl-CoA, the first step in the transport of long-chain fatty acids from the cytosol into the mitochondrial matrix, where they are oxidized. ${ }^{28,38)}$

In conclusion, we demonstrated that gypenosides treatment reduced the liver fatty acid accumulation in NAFLD rat models by reducing the expression of the key transcriptional factors SREBP-1c and ChREBP as well as the lipogenic enzymes ACCase, malonyl CoA, and SCD-1. Meanwhile, it upregulated the expression of the lipogenic enzyme CPT-1, which is involved in fatty acid oxidation in liver tissues. Therefore, gypenosides have a therapeutic effect on NASH through regulating hepatic lipogenesis and fatty acid oxidation. Though the potential downstream regulatory pathway of gypenosides in NAFLD is unclear, we thought that by regulating fatty acid metabolism and inhibiting fatty acid production, gypenosides may lead to a decrease of TG synthesis via reducing the material for TG synthesis, thus causing increased transport out of the liver. As the source of gypenosides is widespread and its quality can be controlled, it is expected that gypenosides could be an effective anti-NASH drug in the clinic, after the pharmacodynamic effects and mechanism are further elucidated.

Acknowledgments This study was supported by Grants from the National Natural Science Foundation of China (81503404), Zhejiang Provincial Natural Science Foundation (LY15H270002), and Zhejiang Provincial Construction Project of Province and City Co-building Key Disciplines (2016-7).

Conflict of Interest The authors declare no conflict of interest.

\section{REFERENCES}

1) Loomba R, Sanyal AJ. The global NAFLD epidemic. Nat. Rev. Gastroenterol. Hepatol., 10, 686-690 (2013).

2) Bellentani S, Scaglioni F, Marino M, Bedogni G. Epidemiology of non-alcoholic fatty liver disease. Dig. Dis., 28, 155-161 (2010).

3) Bhala N, Jouness RI, Bugianesi E. Epidemiology and natural history of patients with NAFLD. Curr. Pharm. Des., 19, 5169-5176 (2013).

4) Fan JG, Zhu J, Li XJ, Chen L, Li L, Dai F, Li F, Chen SY. Prevalence of and risk factors for fatty liver in a general population of Shanghai, China. J. Hepatol., 43, 508-514 (2005).

5) Jimba $S$, Nakagami T, Takahashi M, Wakamatsu T, Hirota Y, Iwamoto Y, Wasada T. Prevalence of non-alcoholic fatty liver disease and its association with impaired glucose metabolism in Japanese adults. Diabet. Med., 22, 1141-1145 (2005).

6) Kwon YM, Oh SW, Hwang SS, Lee C, Kwon H, Chung GE. Association of nonalcoholic fatty liver disease with components of metabolic syndrome according to body mass index in Korean adults. Am. J. Gastroenterol., 107, 1852-1858 (2012).

7) Milic S, Mikolasevic I, Krznaric-Zrnic I, Stanic M, Poropat G, Stimac D, Vlahovic-Palcevski V, Orlic L. Nonalcoholic steatohepatitis: emerging targeted therapies to optimize treatment options. Drug Des. Devel. Ther., 9, 4835-4845 (2015).

8) Wong RJ, Cheung R, Ahmed A. Nonalcoholic steatohepatitis is the most rapidly growing indication for liver transplantation in patients with hepatocellular carcinoma in the U.S. Hepatology, 59, 2188-2195 (2014).

9) Ramadori P, Drescher H, Erschfeld S, Schumacher F, Berger C, Fragoulis A, Schenkel J, Kensler TW, Wruck CJ, Trautwein C, Kroy DC, Streetz KL. Hepatocyte-specific Keap1 deletion reduces liver steatosis but not inflammation during non-alcoholic steatohepatitis development. Free Radic. Biol. Med., 91, 114-126 (2016).

10) Lee JJ, Lambert JE, Hovhannisyan Y, Ramos-Roman MA, Trombold JR, Wagner DA, Parks EJ. Palmitoleic acid is elevated in fatty liver disease and reflects hepatic lipogenesis. Am. J. Clin. Nutr., 101, 34-43 (2015).

11) Fromenty B, Pessayre D. Inhibition of mitochondrial beta-oxidation as a mechanism of hepatotoxicity. Pharmacol. Ther., 67, 101-154 (1995).

12) Okamoto $T$, Koda M, Miyoshi K, Onoyama $T$, Kishina M, Matono 
T, Sugihara T, Hosho K, Okano J, Isomoto H, Murawaki Y. Antifibrotic effects of ambrisentan, an endothelin-A receptor antagonist, in a non-alcoholic steatohepatitis mouse model. World J. Hepatol., 8, 933-941 (2016).

13) Pascale A, Pais R, Ratziu V. An overview of nonalcoholic steatohepatitis: past, present and future directions. J. Gastrointestin. Liver Dis., 19, 415-423 (2010).

14) Xia J, Xin L, Zhu W, Li L, Li C, Wang Y, Mu Y, Yang S, Li K. Characterization of long non-coding RNA transcriptome in highenergy diet induced nonalcoholic steatohepatitis minipigs. Sci. Rep., 6, 30709 (2016).

15) Kong L, Wang X, Zhang K, Yuan W, Yang Q, Fan J, Wang P, Liu Q. Gypenosides Synergistically Enhances the Anti-Tumor Effect of 5-Fluorouracil on Colorectal Cancer in vitro and in vivo: A Role for Oxidative Stress-Mediated DNA Damage and p53 Activation. PLoS ONE, 10, e0137888 (2015).

16) Quan Y, Yang Y, Wang H, Shu B, Gong QH, Qian M. Gypenosides attenuate cholesterol-induced DNA damage by inhibiting the production of reactive oxygen species in human umbilical vein endothelial cells. Mol. Med. Rep., 11, 2845-2851 (2015).

17) Ding WJ, Wang Y, Fan JG. Regulation of adipokines by polyunsaturated fatty acids in a rat model of non-alcoholic steatohepatitis. Arch. Iran Med., 17, 563-568 (2014).

18) Torres DM, Jones FJ, Shaw JC, Williams CD, Ward JA, Harrison SA. Rosiglitazone versus rosiglitazone and metformin versus rosiglitazone and losartan in the treatment of nonalcoholic steatohepatitis in humans: a 12-month randomized, prospective, open- label trial. Hepatology, 54, 1631-1639 (2011).

19) Tan Y, Lao W, Xiao L, Wang Z, Xiao W, Kamal MA, Seale JP, Qu $X$. Managing the combination of nonalcoholic Fatty liver disease and metabolic syndrome with chinese herbal extracts in highfat-diet fed rats. Evid. Based Complement. Alternat. Med., 2013, 306738 (2013).

20) Jian-gao F, Chinese Liver Disease A. Guidelines for management of nonalcoholic fatty liver disease: an updated and revised edition. Zhonghua Gan Zang Bing Za Zhi, 18, 163-166 (2010).

21) Chen SD, Li J, Zhou HH, Lin MT, Liu YH, Zhang YM, Zhao ZX, Li GH, Liu J. Study on effects of zhi zi (fructus gardeniae) on nonalcoholic fatty liver disease in the rat. J. Tradit. Chin. Med., 32, 82-86 (2012).

22) Saito T, Misawa K, Kawata S. Fatty liver and non-alcoholic steatohepatitis. Intern. Med., 46, 101-103 (2007).

23) Li Y, Jadhav K, Zhang Y. Bile acid receptors in non-alcoholic fatty liver disease. Biochem. Pharmacol., 86, 1517-1524 (2013).

24) Knebel B, Lehr S, Hartwig S, Haas J, Kaber G, Dicken HD, Susanto F, Bohne L, Jacob S, Nitzgen U, Passlack W, Muller-Wieland D, Kotzka J. Phosphorylation of sterol regulatory element-binding protein (SREBP)-1c by p38 kinases, ERK and JNK influences lipid metabolism and the secretome of human liver cell line HepG2. Arch. Physiol. Biochem., 120, 216-227 (2014).

25) Kotzka J, Knebel B, Janssen OE, Schaefer JR, Soufi M, Jacob S, Nitzgen U, Muller-Wieland D. Identification of a gene variant in the master regulator of lipid metabolism SREBP-1 in a family with a novel form of severe combined hypolipidemia. Atherosclerosis, 218, 134-143 (2011).

26) Wójcicka G, Jamroz-Wiśniewska A, Horoszewicz K, Bełtowski J. Liver X receptors (LXRs). Part I: structure, function, regulation of activity, and role in lipid metabolism. Postepy. Hig. Med Dosw. (Online), 61, 736-759 (2007).

27) Nakamuta M, Fujino T, Yada R, Yada M, Yasutake $K$, Yoshimoto $T$, Harada N, Higuchi N, Kato M, Kohjima M, Taketomi A, Maehara Y, Nakashima M, Kotoh K, Enjoji M. Impact of cholesterol metabolism and the LXRalpha-SREBP-1c pathway on nonalcoholic fatty liver disease. Int. J. Mol. Med., 23, 603-608 (2009).

28) Musso G, Gambino R, Cassader M. Recent insights into hepatic lipid metabolism in non-alcoholic fatty liver disease (NAFLD). Prog. Lipid Res., 48, 1-26 (2009).

29) Strable MS, Ntambi JM. Genetic control of de novo lipogenesis: role in diet-induced obesity. Crit. Rev. Biochem. Mol. Biol., 45, 199-214 (2010).

30) Chen J, Jing G, Xu G, Shalev A. Thioredoxin-interacting protein stimulates its own expression via a positive feedback loop. Mol. Endocrinol., 28, 674-680 (2014).

31) Cohen P, Miyazaki M, Socci ND, Hagge-Greenberg A, Liedtke W, Soukas AA, Sharma R, Hudgins LC, Ntambi JM, Friedman JM. Role for stearoyl-CoA desaturase-1 in leptin-mediated weight loss. Science, 297, 240-243 (2002).

32) Salie MJ, Zhang N, Lancikova V, Xu D, Thelen JJ. A Family of Negative Regulators Targets the Committed Step of De novo Fatty Acid Biosynthesis. Plant Cell, 28, 2312-2325 (2016).

33) Chen J, Wu W, Fu Y, Yu S, Cui D, Zhao M, Du Y, Li J, Li X. Increased expression of fatty acid synthase and acetyl-CoA carboxylase in the prefrontal cortex and cerebellum in the valproic acid model of autism. Exp. Ther. Med., 12, 1293-1298 (2016).

34) Munday MR. Regulation of mammalian acetyl-CoA carboxylase. Biochem. Soc. Trans., 30, 1059-1064 (2002).

35) Ntambi JM, Miyazaki M. Regulation of stearoyl-CoA desaturases and role in metabolism. Prog. Lipid Res., 43, 91-104 (2004).

36) Shimano H. Sterol regulatory element-binding proteins (SREBPs): transcriptional regulators of lipid synthetic genes. Prog. Lipid Res., 40, 439-452 (2001).

37) Ntambi JM, Miyazaki M, Dobrzyn A. Regulation of stearoyl-CoA desaturase expression. Lipids, 39, 1061-1065 (2004).

38) Suzuki H, Kawarabayasi Y, Kondo J, Abe T, Nishikawa K, Kimura $\mathrm{S}$, Hashimoto T, Yamamoto T. Structure and regulation of rat longchain acyl-CoA synthetase. J. Biol. Chem., 265, 8681-8685 (1990). 